\title{
Evaluation of a new selective medium for the isolation of Corynebacterium urealyticum
}

\author{
J. ZAPARDIEL, EVA NIETO and F. SORIANO \\ Department of Medical Microbiology, Fundación Jimenez Díaz, Avenida de Reyes Católicos 2, 28040 Madrid, \\ Spain
}

\begin{abstract}
A new selective medium (CBU agar) was compared with blood agar (BA) medium for primary isolation of Corynebacterium urealyticum from urine and skin samples of hospitalised patients. Overall, the CBU agar detected $C$. urealyticum in $14(4.6 \%)$ of 302 urine samples and the $B A$ medium detected the organism in four $(1.3 \%)$, but most cultures which were positive only on $\mathrm{CBU}$ agar had $<10^{4} \mathrm{cfu} / \mathrm{ml}$. Six strains of $C$. urealyticum were isolated from 60 skin samples with CBU agar, whereas none was detected with BA. Although most skin samples had heavy inocula, the selective agar facilitated the recognition of low colony counts $(\leqslant 10 \mathrm{cfu} / \mathrm{plate})$ of $C$. urealyticum by reducing the growth of competing flora. Challenge of the selective medium with reference and clinical strains showed that CBU agar was inhibitory for gram-negative bacteria and reduced the gram-positive flora, allowing the growth of $C$. urealyticum strains. The new selective medium appears to be a useful epidemiological tool to study urinary and skin colonisation by $C$. urealyticum.
\end{abstract}

\section{Introduction}

In the last two decades, Corynebacterium urealyticum, a urea-splitting and slow-growing bacterium, has been documented as an opportunist human pathogen, mainly in urinary tract infections [1-7]. Its fastidious nature and slow growth explain the difficulties encountered in its isolation from 24-h cultures of urine samples or from mixed cultures with fast-growing organisms. Two selective or semi-selective media have been developed to enhance the isolation of $C$. urealyticum from urine samples [8, 9]. Although the selective media improved the isolation of multiresistant $C$. urealyticum strains usually involved in human infections [8-11], occasionally, some $\beta$-lactam-susceptible strains were isolated only with the non-selective media $[8,11]$. The evidence for non-multiresistant isolates of $C$. urealyticum in clinical samples $[7,8,11-13]$ and an interest in understanding the epidemiology of such bacteria, prompted the development of a new selective medium for the isolation of $C$. urealyticum. Although this medium has been used previously to isolate $C$. urealyticum from urine [14] and environmental samples [15], the selectivity, quantitative recovery and colonial morphology have not been documented. This study describes the growth, selectivity studies and the performance of the selective medium to isolate multi-

Received 25 March 1997; accepted 23 May 1997. Corresponding author: Dr F. Soriano. ple-antibiotic resistant and non-multiresistant strains of C. urealyticum from urine and skin samples.

\section{Materials and methods}

\section{Culture media}

The novel selective medium (CBU agar) has the following composition (/L): Heart Infusion Agar (Difco Laboratories, Detroit, MI, USA) $40 \mathrm{~g}$; L-cysteine hydrochloride (Merck, Germany) $0.128 \mathrm{~g}$; phenol red (Merck) $0.01 \mathrm{~g}$; urea (Merck) $20 \mathrm{~g}$; glucose (Scharlau, Barcelona, Spain) $10 \mathrm{~g}$; polisorbate 80 (Tween 80; Difco) $10 \mathrm{ml}$; fosfomycin (Cepa SA, Madrid, Spain) $50 \mathrm{mg}$; polymixin B (Sigma) $2500 \mathrm{IU}$; aztreonam (Squibb, Barcelona, Spain) $16 \mathrm{mg}$; and amphotericin B (Squibb) $2 \mathrm{mg}$.

A CBU agar medium without antibiotics (CBUw/a) was prepared as a control for growth and selectivity studies. A heart infusion agar (Difco) with sheep blood 5\% (BA) was used as a control to culture the clinical samples. Inocula for growth and selectivity studies were performed in Brain Heart Infusion Broth (Difco).

\section{Antimicrobial susceptibility tests}

To challenge the antimicrobial agents included in the selective medium, the susceptibilities of one multi- 
resistant reference strain (C. urealyticum ATCC $43042^{\mathrm{T}}$ ) and six non-multiresistant stock collection strains to ampicillin, aztreonam, fosfomycin and polymixin B were tested. A microdilution method was performed in cation-supplemented Mueller-Hinton Broth (Difco) containing lysed horse blood 3\%, following the guidelines described by the NCCLS for streptococci [16].

The antimicrobial susceptibility of $C$. urealyticum strains from the urine and skin samples was studied by an agar-diffusion method, as reported previously $[17,18]$.

Growth studies and quantitative recovery of $C$. urealyticum in the selective medium

Overnight broth cultures of two reference strains and five non-multiresistant stock collection strains of $C$. urealyticum (Table 1) were adjusted to 0.5 MacFarland turbidity and eight serial ( 1 in 10) dilutions were made with a phosphate buffer. Samples of $0.1 \mathrm{ml}$ were plated in duplicate on CBU agar as well as on to CBUw/a agar. The inocula were spread over the entire plate area to obtain isolated colonies. Plates were incubated aerobically at $35^{\circ} \mathrm{C}$ for $72 \mathrm{~h}$. They were examined daily and the following data were recorded: length of time for growth to appear, number of colonies, size and morphology, colour changes in the medium and Tween hydrolysis. To test the quantitative recovery of $C$. urealyticum in the $\mathrm{CBU}$ agar, colony counts were made from the $10^{5}$ dilution.

\section{Selectivity}

Quantitative and qualitative tests were performed to check the selectivity of the CBU agar. Reference strains and clinical isolates of species usually involved in urinary tract infections or belonging to healthy skin flora and some environmental or animal strains of diphtheroids were selected.

Firstly, a quantitative analysis was performed by inoculating $\mathrm{CBU}$ agar and $\mathrm{CBUw} / \mathrm{a}$ agar plates with a defined inoculum of $(0.5-1) \times 10^{3} \mathrm{cfu} / \mathrm{ml}$. The challenged strains were $C$. jeikeium LA-89, Staphylococcus aureus ATCC 25923, Enterococcus faecalis ATCC 29212, Escherichia coli ATCC 25922, Klebsiella pneumoniae ATCC 10028, Proteus mirabilis CCI, Pseudomonas aeruginosa ATCC 27835, S. epidermidis ATCC 12822, Coryneform CDC group F CDC-F5863 and Candida albicans ATCC 90028. In addition, the colonial morphology of control strains on the CBU agar base without antibiotics was compared with those of $C$. urealyticum on CBU agar.

Secondly, to evaluate the selectivity of the medium with a higher inoculum and a large number of strains from different genera (Table 2), inocula of $c .10^{4}$ $\mathrm{cfu} /$ spot were plated on to a selective CBU agar and non-selective blood agar medium with a Steers type device and were incubated aerobically up to $72 \mathrm{~h}$ at $35^{\circ} \mathrm{C}$ and examined daily for growth.

\section{Clinical samples}

A total of 302 urine samples from 282 patients, hospitalised in different wards of the hospital was cultured to assess the reliability of CBU agar in the detection of $C$. urealyticum bacteriuria. Mid-stream urine samples were plated with a calibrated loop $(0.05 \mathrm{ml})$ on to a non-selective $\mathrm{BA}$ and on a selective CBU agar.

The performance of the selective agar in the detection of $C$. urealyticum from healthy skin was also studied. Twenty in-patients were sampled at admission or during the first $24 \mathrm{~h}$ of hospitalisation. Skin surfaces of c. $4 \mathrm{~cm}^{2}$ in the axilla, groin and anterior abdominal wall were sampled following a swab-rinse sampling method, as described previously [19]. Sixty skin samples were cultured quantitatively by inoculating three serialy diluted $(1$ in 10$)$ and one undiluted sample $(0.1 \mathrm{ml})$ on to plates of CBU agar and BA.

Both CBU agar and BA plates were incubated at $37^{\circ} \mathrm{C}$ for $72 \mathrm{~h}$. Colonies with typical C. urealyticum morphology and Gram's stain appearance and positive catalase reaction were tested for urease activity, glucose acidification and nitrate reduction. Only isolates that were urease positive, glucose and nitrate negative, were further identified to species level by standard methods [17, 20].

\section{Results}

All seven $C$. urealyticum strains tested by the microdilution assay were resistant to fosfomycin (MICs $>128 \mu \mathrm{g} / \mathrm{ml}$ ), aztreonam (MICs $>128 \mu \mathrm{g} / \mathrm{ml}$ ) and polymixin B (MICs $2-8 \mu \mathrm{g} / \mathrm{ml}$ ). The reference strain and one non-multiresistant strain (NMS-1) were also resistant to ampicillin, with the remainder being susceptible (MICs $\leqslant 0.5 \mu \mathrm{g} / \mathrm{ml}$ ).

In the growth studies, all stock and reference strains of $C$. urealyticum grew on the selective and nonselective media (Table 1). The most typical morphology of $C$. urealyticum in CBU agar was seen after incubation for $48-72 \mathrm{~h}$, when the colonies were pale yellow, circular, $0.5-1.5 \mathrm{~mm}$ in diameter, opaque, glistening, smooth, convex, with entire edges and then, the whole medium was usually red, especially with high inocula. Occasionally, C. urealyticum strains formed a white halo surrounding the colonies, which may be caused by Tween 80 hydrolysis.

In the quantitative selectivity test performed with stock and reference strains, C. jeikeium LA-89 and Coryneform group F CDC-F5863 grew after $48 \mathrm{~h}$ on 
Table 1. Quantitative recovery of C. urealyticum on CBU agar

\begin{tabular}{|c|c|c|c|c|c|c|}
\hline \multirow[b]{2}{*}{ Strains* } & \multirow[b]{2}{*}{ Data at } & \multicolumn{2}{|c|}{ CBU w/a agar } & \multicolumn{2}{|c|}{ CBU agar } & \multirow[b]{2}{*}{$\begin{array}{l}\text { Percentage } \\
\text { recovery }^{\dagger}\end{array}$} \\
\hline & & $\begin{array}{l}\text { Colony size } \\
(\mathrm{mm})\end{array}$ & $\begin{array}{c}\text { Number of } \\
\text { colonies }\end{array}$ & $\begin{array}{l}\text { Colony size } \\
(\mathrm{mm})\end{array}$ & $\begin{array}{l}\text { Number of } \\
\text { colonies }\end{array}$ & \\
\hline ATCC 43042 & $\begin{array}{l}24 \mathrm{~h} \\
48 \mathrm{~h} \\
72 \mathrm{~h}\end{array}$ & $\begin{array}{c}0 \\
0.8-1 \\
1-2\end{array}$ & $\begin{array}{r}0 \\
115 \\
115\end{array}$ & $\begin{array}{c}0 \\
1 \\
1.5-2\end{array}$ & $\begin{array}{r}0 \\
99 \\
99\end{array}$ & $\begin{array}{r}0 \\
86 \\
86\end{array}$ \\
\hline ATCC 43043 & $\begin{array}{l}24 \mathrm{~h} \\
48 \mathrm{~h} \\
72 \mathrm{~h}\end{array}$ & $\begin{array}{r}0.1-0.3 \\
1-1.5 \\
1-1.5\end{array}$ & $\begin{array}{l}50 \\
50 \\
50\end{array}$ & $\begin{array}{c}0 \\
1-1.5 \\
1-1.5\end{array}$ & $\begin{array}{r}0 \\
25 \\
25\end{array}$ & $\begin{array}{r}0 \\
50 \\
50\end{array}$ \\
\hline NMS-1 & $\begin{array}{l}24 \mathrm{~h} \\
48 \mathrm{~h} \\
72 \mathrm{~h}\end{array}$ & $\begin{array}{c}0 \\
0.8-2 \\
0.8-2\end{array}$ & $\begin{array}{r}0 \\
25 \\
25\end{array}$ & $\begin{array}{c}0 \\
0.1-1.5 \\
0.1-1.5\end{array}$ & $\begin{array}{r}0 \\
25 \\
25\end{array}$ & $\begin{array}{r}0 \\
100 \\
100\end{array}$ \\
\hline NMS-2 & $\begin{array}{l}24 \mathrm{~h} \\
48 \mathrm{~h} \\
72 \mathrm{~h}\end{array}$ & $\begin{array}{c}0.1-0.3 \\
1-1.5 \\
1.5-2\end{array}$ & $\begin{array}{l}15 \\
55 \\
55\end{array}$ & $\begin{array}{c}0 \\
0.1-1.5 \\
0.5-1.5\end{array}$ & $\begin{array}{r}0 \\
50 \\
50\end{array}$ & $\begin{array}{c}0 \\
90.9 \\
90.9\end{array}$ \\
\hline NMS-3 & $\begin{array}{l}24 \mathrm{~h} \\
48 \mathrm{~h} \\
72 \mathrm{~h}\end{array}$ & $\begin{array}{r}0.1 \\
0.1-1 \\
0.1-1\end{array}$ & $\begin{array}{l}25 \\
25 \\
25\end{array}$ & $\begin{array}{c}0 \\
0.5-1 \\
0.5-1\end{array}$ & $\begin{array}{r}0 \\
25 \\
25\end{array}$ & $\begin{array}{r}0 \\
100 \\
100\end{array}$ \\
\hline NMS-4 & $\begin{array}{l}24 \mathrm{~h} \\
48 \mathrm{~h} \\
72 \mathrm{~h}\end{array}$ & $\begin{array}{c}0 \\
0.8-1.2 \\
0.8-1.2\end{array}$ & $\begin{array}{r}0 \\
50 \\
50\end{array}$ & $\begin{array}{c}0 \\
0.3-1 \\
0.3-1.2\end{array}$ & $\begin{array}{r}0 \\
50 \\
50\end{array}$ & $\begin{array}{r}0 \\
100 \\
100\end{array}$ \\
\hline NMS-5 & $\begin{array}{l}24 \mathrm{~h} \\
48 \mathrm{~h} \\
72 \mathrm{~h}\end{array}$ & $\begin{array}{r}0.1-0.3 \\
1-1.5 \\
1-1.5\end{array}$ & $\begin{array}{l}30 \\
30 \\
30\end{array}$ & $\begin{array}{c}0 \\
1-1.2 \\
1-1.5\end{array}$ & $\begin{array}{r}0 \\
50 \\
50\end{array}$ & $\begin{array}{r}0 \\
166 \\
166\end{array}$ \\
\hline
\end{tabular}

${ }^{*} \mathrm{NMS}=$ Non-multiresistant stock collection strains of C. urealyticum.

†Percentage of quantitative recovery of $C$. urealyticum in CBU agar, compared with CBUw/a agar colony counts.

CBU agar and CBUw/a agar. The other reference strains grew on CBUw/a agar after incubation for $24 \mathrm{~h}$, but no growth was detected on the CBU agar after incubation for $72 \mathrm{~h}$. Staphylococci, enterococci, enterobacteria, $P$. aeruginosa and $C$. albicans had different colonial morphologies on CBUw/a agar and their morphotypes were easily distinguished from $C$. urealyticum. The results of the qualitative selectivity test are shown in Table 2.

C. urealyticum was detected in $14(4.6 \%)$ of 302 urine cultures with CBU agar, but only four $(1.3 \%)$ of those cultures were detected in both media. No $C$. urealyticum isolate was in pure culture and all except two had colony counts $<10^{5} \mathrm{cfu} / \mathrm{ml}$.

C. urealyticum and gram-negative bacilli were isolated on the BA plates from four urine samples. The rest of the samples harbouring $C$. urealyticum yielded mixed microbial flora (eight samples) or gram-negative bacilli (two samples) on the BA plates and $C$. urealyticum was missed. Only six samples yielded $C$. urealyticum in pure culture in CBU agar. Otherwise, eight mixed cultures with gram-positive cocci (two samples) or with other gram-positive bacilli (six samples) were obtained.

Seventy-one urine samples not harbouring $C$. urealyticum had positive cultures on the CBU agar plate. Gram-positive bacilli $(n=55)$, staphylococci $(n=21)$, yeasts $(n=9)$ and gram-negative bacilli $(n=1)$ were isolated. Thirteen $C$. urealyticum strains were multiresistant and one strain was $\beta$-lactam-resistant.
Sixty skin swabs from three different sites of 20 inpatients were inoculated quantitatively on to BA and CBU agar. As most skin samples had heavy inocula, many undiluted samples had abundant growth on BA and CBU agar plates. Micrococci, staphylococci and coryneform bacteria were usually isolated from the skin on the BA plate, but on CBU agar, micrococci or staphylococci were scarcely and occasionally recovered, with coryneform bacteria being the predominant growth. Six strains of $C$. urealyticum were isolated in five samples from five patients with the CBU agar plates, but none was detected on BA. Although heavy growth was present on $\mathrm{CBU}$ agar, $C$. urealyticum had $\leqslant 10$ colonies per plate in all but one of the positive cultures. Several antibiotypes were present among isolates: multiresistant, $\beta$-lactam-resistant and $\beta$-lactam-susceptible.

\section{Discussion}

C. urealyticum causes nosocomial infections, mainly urinary tract infections, in immunosuppressed patients with renal transplantation or urological diseases $[1,4]$. The isolation of $C$. urealyticum from clinical samples is difficult because of its fastidious growth, especially in the presence of non-fastidious normal flora. Although prolonged incubation for $48 \mathrm{~h}$ or longer improved the isolation of $C$. urealyticum on nonselective media $[11,7,21]$, the use of selective media enhanced the recovery of multiresistant $C$. urealyticum from urine samples [8-11]. Those selective media contain either $\beta$-lactam antibiotics [8] or clindamycin 
Table 2. Qualitative selectivity test

\begin{tabular}{|c|c|}
\hline Species & $\begin{array}{l}\text { Number of strains } \\
\text { tested/inhibited }\end{array}$ \\
\hline \multicolumn{2}{|l|}{ Coryneform bacteria } \\
\hline Arcanobacterium haemolyticum & $7 / 7$ \\
\hline Actinomyces pyogenes & $4 / 4$ \\
\hline 'C. aquaticum' & $4 / 2$ \\
\hline 'C. bovis' & $2 / 0$ \\
\hline \multicolumn{2}{|l|}{ C. jeikeium } \\
\hline Multiresistant & $36 / 0$ \\
\hline Susceptible & $3 / 0^{*}$ \\
\hline C. minutissimum & $19 / 3$ \\
\hline C. pilosum $/$ C. renale & $4 / 4$ \\
\hline C. pseudodiphtheriticum & $12 / 3^{*}$ \\
\hline C. pseudotuberculosis & $4 / 0$ \\
\hline C. striatum & $11 / 1$ \\
\hline C. ulcerans & $6 / 0$ \\
\hline \multicolumn{2}{|l|}{ C. urealyticum } \\
\hline Multiresistant & $37 / 0^{*}$ \\
\hline Susceptible & $9 / 0^{*}$ \\
\hline C. xerosis & $20 / 2$ \\
\hline Coryneform CDC group A & $8 / 3$ \\
\hline Coryneform CDC group ANF & $6 / 3$ \\
\hline Coryneform CDC group $\mathrm{F}$ & $15 / 5$ \\
\hline Erysipelothrix rhusiopathiae & $7 / 7$ \\
\hline Listeria monocytogenes & $16 / 1$ \\
\hline Oerskovia spp. & $4 / 0$ \\
\hline Rothia dentocariosa & $2 / 2$ \\
\hline Rhodococcus equi & $9 / 0$ \\
\hline Subtotal & $245 / 51(20.8 \%)$ \\
\hline \multicolumn{2}{|l|}{ Gram-negative bacilli } \\
\hline Escherichia coli & $53 / 53$ \\
\hline Acinetobacter spp. & $13 / 13$ \\
\hline Klebsiella pneumoniae & $15 / 15$ \\
\hline Citrobacter spp. & $6 / 6$ \\
\hline Enterobacter spp. & $14 / 13^{\dagger}$ \\
\hline Pseudomonas aeruginosa & $17 / 17$ \\
\hline Pseudomomas spp. & $4 / 2^{+}$ \\
\hline Serratia marcescens & $6 / 6$ \\
\hline Subtotal & $128 / 125(97.6 \%)$ \\
\hline \multicolumn{2}{|l|}{ Gram-positive cocci } \\
\hline Enterococcus spp. & $35 / 26$ \\
\hline$S$. aureus & $18 / 3$ \\
\hline $\begin{array}{l}\text { Staphylococcus spp. } \\
\text { (coagulase negative) }\end{array}$ & $6 / 2$ \\
\hline Subtotal & $59 / 31(52.5 \%)$ \\
\hline
\end{tabular}

plus nalidixic acid [9] to inhibit or reduce other organisms present in the sample. Occasionally, urine samples containing $\beta$-lactam-susceptible isolates of $C$. urealyticum were missed when plated on to $\beta$-lactamcontaining media $[8,11]$ and several strains susceptible to $\beta$-lactam $[12,22]$ or clindamycin $[22-24]$ have been reported. As these antibiotic supplements may represent a negative selection for fully susceptible strains, a different antibiotic supplement consisting of fosfomycin, aztreonam, polymyxin B and amphotericin B was selected. Fosfomycin has been used successfully in selective media for multiresistant corynebacteria $[8$, $25]$, so the 'in-vitro' inactivity against a multiresistant reference strain and six susceptible clinical stock collection strains was confirmed by a microdilution method. Aztreonam was selected because of its activity against gram-negative bacilli and all seven strains tested by microdilution were resistant $(>128 \mathrm{mg} / \mathrm{L})$, as previously reported for multiresistant strains [23]. Polymyxin B and amphotericin B were added at concentrations used in several selective media [9].

CBU agar is a lipid-enriched peptone-based agar like those previously described for the primary isolation of C. jeikeium $[8,25]$ and $C$. urealyticum $[8,9]$. CBU agar is also made into a differential medium by the addition of urea, glucose and phenol red. $C$. urealyticum is a urea-splitting, asaccharolytic bacterium forming typical colonies surrounded by a red halo on CBU agar. Because of its strong urease activity, when $>10$ colonies grew, the whole plate was pink and only the colonial morphology of $C$. urealyticum allowed its recognition. Other urease-producing bacteria isolated on the medium grew faster or were glucose fermenting and no clear red halo was seen. Although CBU agar allowed the isolation of antibiotic-susceptible and multiresistant strains of C. urealyticum, growth was slower on the selective medium as compared with the agar base without antibiotics. The final number, size and morphology of colonies was similar on the selective and non-selective media. The most typical colonial morphology was seen after incubation for $48-72 \mathrm{~h}$, so we recommend incubation for at least $48 \mathrm{~h}$ for plates inoculated with clinical samples or $72 \mathrm{~h}$ for samples cultured for epidemiological purposes.

The selectivity of CBU agar was excellent against gram-negative bacilli, not only in the selectivity studies with reference or clinical strains, but also for the urine samples cultured. Other isolates that rarely grew on CBU agar included multiresistant enterobacteria or pseudomonas and were easily recognised by their colonial morphology or absence of urease. Although the selectivity of CBU agar to gram-positive cocci was good for reference strains, it was less than expected for clinical isolates tested by the qualitative method. Furthermore, there was poor selectivity against micrococci, staphylococci or enterococci when plated with the urine and skin samples. Again, it was possible to distinguish $C$. urealyticum isolates because their urease and colonial morphology differed. Several coryneform bacteria other than $C$. urealyticum grew on CBU agar, most were non-urease producing and formed colonies different to those of $C$. urealyticum.

The selective media enhanced the recovery of $C$. urealyticum [8-11] but their utility in the practical management of patients is controversial $[9,11,14$, 21]. Although the use of selective media for $C$. urealyticum to culture urine samples from patients at high risk has been sustained $[9,14]$, most isolates of C. urealyticum grown only from selective media had no clinical relevance $[8,10,11,21]$. CBU agar also improved the primary isolation of $C$. urealyticum in urine samples from unselected in-patients, yielding a 3.5-fold increase $(1.3-4.6 \%)$, as has been reported for other selective media $[8,10,11,14,21]$. Garcia-Bravo 
et al. reported a two-fold increase in recovery of $C$. urealyticum from urine samples of renal transplant patients with this selective medium [14]. The clinical significance of the urine isolates in the present study was not analysed, but all $C$. urealyticum strains were in mixed culture and only two cultures had colony counts $\geqslant 10^{5} \mathrm{cfu} / \mathrm{ml}$ of $C$. urealyticum and Pseudomonas aeruginosa. Therefore, we do not recommend the selective medium for routine urine cultures, but just for epidemiological surveys.

Multiresistant strains of $C$. urealyticum have been isolated from the healthy skin of infected and colonised patients by plating the skin samples on to $\beta$-lactam-containing selective media $[4,8,26]$. As the presence of antibiotic-susceptible strains would be missed with such media, the reliability of CBU agar for the isolation of non-multiresistant skin strains was tested. The selective agar was plated with skin swabs from patients at their first day of hospitalisation. As has been shown with the growth studies and clinical samples, the selective medium had excellent sensitivity for the isolation of $C$. urealyticum ( $\leqslant 10$ colonies), although a heavy growth was present on the plate. The CBU agar was effective in isolating multiresistant and antibiotic-susceptible strains of $C$. urealyticum from skin samples. Finally, CBU agar has been used successfully in a prospective study of environmental contamination by $C$. urealyticum in a hospital, showing its reliability in isolating the bacteria from air and surface samples [15].

In conclusion, $\mathrm{CBU}$ agar supports the growth of $C$. urealyticum, including those isolates which could be inhibited in other selective media, facilitates isolation by reducing or inhibiting the growth of competing flora, and produces typical colonies. This selective medium appears to be an excellent epidemiological tool for the isolation of $C$. urealyticum from human or environmental samples.

This work was supported by grants from the Fondo de Investigaciones Sanitarias de la Seguridad Social (90/233) and Fundación Conchita Rábago.

\section{References}

1. Aguado JM, Salto E, Morales JM et al. Corynebacterium urealyticum: a new and threatening pathogen for the renal transplant patient. Transplant Proc 1993; 25: 1493-1494.

2. Coyle MB, Lipsky BA. Coryneform bacteria in infectious diseases: clinical and laboratory aspects. Clin Microbiol Rev 1990; 3: 227-246.

3. Soriano F, Fernandez-Roblas R. Infections caused by antibioticresistant Corynebacterium group D2. Eur $J$ Clin Microbiol Infect Dis 1988; 7: 337-341.

4. Soriano F, Aguado JM, Ponte MC, Fernandez-Roblas R, Rodriguez-Tudela $\mathrm{J}$. Urinary tract infection caused by Corynebacterium group D2: report of 82 cases and review. Rev Infect Dis 1990; 12: 1019-1034.

5. Soriano F, Ponte C, Ruiz P, Zapardiel J. Non-urinary tract infections caused by multiply antibiotic-resistant Corynebacterium urealyticum. Clin Infect Dis 1993; 17: 890-891.
6. Wood CA, Pepe R. Bacteremia in a patient with non-urinary tract infection due to Corynebacterium urealyticum. Clin Infect Dis 1994; 19: 367-368.

7. Zapardiel J, Nieto $\mathrm{E}$, Soriano $\mathrm{F}$. Urinary tract infections by $\beta$ lactam-sensitive Corynebacterium urealyticum strains. Eur $J$ Clin Microbiol Infect Dis 1997; 16: 174-176.

8. De Briel D, Lang JC, Rougeron G, Chabot P, Le Faou A. Multiresistant corynebacteria in bacteriuria: a comparative study of the role of Corynebacterium group D2 and Corynebacterium jeikeium. J Hosp Infect 1991; 17: 35-43.

9. Muñoz JL, Nebreda T, Garcia-Rodriguez JA. [Development of a selective differential medium for the isolation of Corynebacterium urealyticum (group D-2).] Desarrollo de un medio selectivo diferencial para el aislamiento de Corynebacterium urealyticum (grupo D-2). Enferm Infecc Microbiol Clin 1994; 12: $246-250$.

10. Ryan M, Murray PR. Prevalence of Corynebacterium urealyticum in urine specimens collected at a university-affiliated medical center. J Clin Microbiol 1994; 32: 1395-1396.

11. Walkden D, Klugmann KP, Vally $S$, Naidoo P. Urinary tract infection with Corynebacterium urealyticum in South Africa. Eur J Clin Microbiol Infect Dis 1993; 12: 18-24.

12. Jacobs NF, Perlino CA. "Diphtheroid" pneumonia. South Med $J$ 1979; 72: 475-476.

13. Riegel P, Grimont PAD, De Briel D et al. Corynebacterium group D2 ("Corynebacterium urealyticum") constitutes a new genomic species. Res Microbiol 1992; 143: 307-313.

14. Garcia-Bravo M, Aguado JM, Morales JM, Noriega AR. Influence of external factors in resistance of Corynebacterium urealyticum to antimicrobial agents. Antimicrob Agents Chemother 1996; 40: 497-499.

15. Nieto E, Zapardiel J, Soriano F. Environmental contamination by Corynebacterium urealyticum in a teaching hospital. $J$ Hosp Infect 1996; 32: 78-79.

16. National Committee for Clinical Laboratory Standards. Methods for dilution antimicrobial susceptibility tests for bacteria that grow aerobically. Approved Standard M7-A3. Vilanova, PA, National Committee for Clinical Laboratory Standards. 1993.

17. Clarridge JE, Spiegel CA. Corynebacterium and miscellaneous irregular Gram-positive rods, Erysipelothrix, and Gardnerella. In: Murray PR, Baron EJ, Pfaller MA, Tenover FC, Yolken RH (eds) Manual of clinical microbiology, 6th edn. Washington, DC, ASM Press. 1995: 357-378.

18. Van Bosterhaut B, Claeys G, Gigi J, Wauters G. Isolation of Corynebacterium group D2 from clinical specimens. Eur $J$ Clin Microbiol Infect Dis 1987; 6: 418-419.

19. Larson EL, McGinley KJ, Leyden JJ, Cooley ME, Talbot GH Skin colonization with antibiotic-resistant (JK group) and antibiotic-sensitive lipophilic diphtheroids in hospitalized and normal adults. $J$ Infect Dis 1986; 153: 701-706.

20. Hollis DG, Weaver RE. Gram-positive organisms: a guide to identification. Atlanta, GA, Centers for Diseases Control. 1981.

21. Soriano F, Zapardiel J, Ponte C. Use of selective media for isolating Corynebacterium urealyticum from urine specimens $J$ Clin Microbiol 1994; 32: 2630-2631.

22. Soriano F, Zapardiel J, Nieto E. Antimicrobial susceptibilities of Corynebacterium species and other non-spore-forming gram-positive bacilli to 18 antimicrobial agents. Antimicrob Agents Chemother 1995; 39: 208-214.

23. García-Rodriguez JA, García Sánchez JE, Muñoz-Bellido JL, Nebreda Mayoral T, García Sánchez E, García García I. In vitro activity of 79 antimicrobial agents against Corynebacterium group D2. Antimicrob Agents Chemother 1991; 35: 2140-2143.

24. Soriano F, Ponte C, Santamaria M et al. Corynebacterium group D2 as a cause of alkaline-encrusted cystitis: report of four cases and characterization of the organisms. $J$ Clin Microbiol 1985; 21: 788-792.

25. Wichmann S, Wirsing von Koenig $\mathrm{CH}$, Becker-Boost E, Finger $\mathrm{H}$. Isolation of Corynebacterium group $\mathrm{JK}$ from clinical specimens with a semiselective medium. J Clin Microbiol 1983; 19: 204-206.

26. Soriano F, Rodriquez-Tudela JL, Fernández-Roblas R, Aguado JM, Santamaría M. Skin colonization by Corynebacterium groups D2 and JK in hospitalized patients. J Clin Microbiol 1988; 26: $1878-1880$. 